EPJ Web of Conferences 108, 02006 (2016)

DOI: $10.1051 /$ epjconf/201610802006

(C) Owned by the authors, published by EDP Sciences, 2016

\title{
Decoherence and Entanglement Simulation in a Model of Quantum Neural Network Based on Quantum Dots
}

\author{
Mikhail V. Altaisky ${ }^{1, a}$, Nadezhda N. Zolnikova ${ }^{1, b}$, Natalia E. Kaputkina ${ }^{2, c}$, Victor A. Krylov ${ }^{3, d}$, Yurii \\ E. Lozovik ${ }^{4, e}$, and Nikesh S. Dattani ${ }^{5, f}$ \\ ${ }^{1}$ Space Research Institute RAS, Profsoyuznaya 84/32, Moscow, 117997, Russia \\ ${ }^{2}$ National University of Science and Technology "MISIS", Leninsky prospect 4, Moscow, 119049, Russia \\ ${ }^{3}$ Joint Institute for Nuclear Research, Joliot Curie 6, Dubna, 141980, Russia \\ ${ }^{4}$ Institute of Spectroscopy RAS, Troitsk, Moscow, 142190, Russia \\ ${ }^{5}$ Quantum Chemistry Laboratory, Kyoto University, Kyoto, 606-8502, Japan
}

\begin{abstract}
We present the results of the simulation of a quantum neural network based on quantum dots using numerical method of path integral calculation. In the proposed implementation of the quantum neural network using an array of single-electron quantum dots with dipole-dipole interaction, the coherence is shown to survive up to 0.1 nanosecond in time and up to the liquid nitrogen temperature of $77 \mathrm{~K}$. We study the quantum correlations between the quantum dots by means of calculation of the entanglement of formation in a pair of quantum dots on the GaAs based substrate with dot size of $10^{0} \div 10^{1}$ nanometer and interdot distance of $10^{1} \div 10^{2}$ nanometers order.
\end{abstract}

\section{Introduction}

The idea of the quantum neural network (QNN) was first formulated by S.Kak [1]. Since that time different models of quantum neural networks have been considered, see the reviews [2, 3]. The QNNs have earned tremendous attention recently since Google and NASA's announced their use of D-Wave processors for machine supervised learning and big data classification $[4,5]$. However, the $D$-Wave Systems Inc. industrial implementation of QNN, being a Hopfield network built of SQUIDs, has an operating temperature in $\mathrm{mK}$ range to keep quantum coherence for sufficiently long [6]. The low operating temperature results in high energy consumption of the cooling system $(\approx 7 \mathrm{~kW})$ and diminish the prospects of SQUID-based processors for the on-board artificial intelligence devices [7].

We investigate the prospects of alternative elements for artificial quantum neural network - the quantum dots. Quantum dots (QD) are small conductive regions of semiconductor heterostructures (typically less than hundred nanometers) that contain a precisely controlled number of excess electrons. Controllable QDs are often made on the basis of a two-dimensional electron gas of GaAs based

\footnotetext{
a e-mail: altaisky@mx.iki.rssi.ru

be-mail: nzolnik@iki.rssi.ru

ce-mail: nataly@misis.ru

de-mail: kryman@jinr.ru

e-mail: lozovik@isan.troitsk.ru

fe-mail: dattani.nike@gmail.com
} 
heterostructures. The energy levels of the QDs are precisely controlled by the size of the dot and the strength of the external electric and magnetic fields [8-15]. By arranging the QDs in a regular array on a layer of semiconductor heterostructure one can form a matrix for a quantum register composed of either charge-based or spin-based qubits to be used for quantum computations [16].

Using an array of GaAs, QDs for quantum neural networks were first proposed by E.C. Behrman et al. [17]. Their original idea assumed the use of quantum dot molecules interacting with each other only by means of their shared phonon bath. Within this framework, it would be nearly impossible to control the training of the QNN since manipulating a phonon bath is an arduous task [18]. In this paper we present a more achievable quantum dot based QNN arcitecture, where the QDs interact with each other via the dipole-dipole coupling. We present realistic physical parameters for all couplings, and use a numerically exact Feynman integral calculation to study the time evolution of the phonondamped coherence in a pair of one-electron QDs in such a network.

As a quantitative measure of the coherence we have used the entanglement of formation [19] in a pair of QDs subjected to the phonon environment of the GaAs substrate.

\section{Model and Methods}

\subsection{Model Hamiltonian}

We consider a pair of InGaAs/GaAs quantum dots of 3-4 $\mathrm{nm}$ size, as described in [9], where the excitations interact with their bath of acoustic phonons [20-22]. The quantum dots are assumed to interact to each other by the dipole-dipole interaction $J_{i j}=\mu^{2} /\left(\varepsilon L_{i j}^{3}\right)$, where $\mu=\left\langle\mathrm{X} 0\left|e r_{x}\right| 00\right\rangle$ is the transition dipole moment of the QDs. The mean distance between QDs is assumed to be the triple of the size of the $\operatorname{dot} L \approx 10 \mathrm{~nm}, \varepsilon \approx 10$ is the dimensionless GaAs dielectric constant. The Hamiltonian of such system can be written inthe rotating wave approximation as

$$
H=\sum_{i=1}^{2} \frac{\delta_{i}}{2}\left(\sigma_{z}^{(i)}+1\right)+\sum_{i=1}^{2} \frac{K_{i}}{2} \sigma_{x}^{(i)}+\sum_{i \neq j} J_{i j} \sigma_{+}^{(i)} \sigma_{-}^{(j)}+\sum_{a, i} g_{a} x_{a}\left|X_{i}\right\rangle\left\langle X_{i}\right|+H_{P h},
$$

with $\delta_{i}$ being the detuning of the driving electric field frequency from the $i$-th dot transition; $K_{i}$ is the coupling to an external driving field, $J_{i j}$ is the dipole-dipole coupling between the dots, constructed in analogy to the dipole-dipole interaction of the atoms $[23,24]$. The phonon modes $x_{a}$ are assumed to interact only to the excited states $\left|X_{i}\right\rangle$ [25]. The pseudo-spin operators of the $i^{\text {th }} \mathrm{QD}$ are

$$
\sigma_{z}^{(i)}=\left|\mathrm{X}_{i}\right\rangle\left\langle\mathrm{X}_{i}|-| 0_{i}\right\rangle\left\langle 0_{i}\left|, \quad \sigma_{x}^{(i)}=\right| 0_{i}\right\rangle\left\langle\mathrm{X}_{i}|+| \mathrm{X}_{i}\right\rangle\left\langle 0_{i}\left|, \quad \sigma_{+}^{(i)}=\right| \mathrm{X}_{i}\right\rangle\left\langle 0_{i}\left|, \quad \sigma_{-}^{(i)}=\right| 0_{i}\right\rangle\left\langle\mathrm{X}_{i}\right| .
$$

The free phonon Hamiltonian is

$$
H_{P h}=\sum_{a} \frac{p_{a}^{2}}{2 m_{a}}+\frac{m_{a} \omega_{a}^{2} x_{a}^{2}}{2} .
$$

The phonons in the GaAs substrate are assumed to have the spectral density

$$
J(\omega)=\frac{\pi}{2} \sum_{a} \frac{g_{a}}{m_{a} \omega_{a}} \delta\left(\omega-\omega_{a}\right) \approx \alpha \omega^{3} \exp \left(-\left(\omega / \omega_{c}\right)^{2}\right)
$$

where $\alpha$ is the spectral density constant $w_{c}$ is the cutoff frequency. This form of $J(\omega)$ along with these specific parameters have been used consistently in a plethora of studies, in excellent agreement 
between experiment and the theory of the interaction of a single quantum dot with a phonon bath $[8,9,22,26-28]$.

In our studies we used the quasi-adiabatic propagator path integral (QUAPI) technique $[29,30]$ for the solution of the von Neumann equation for the density matrix $\rho(t)$, which describes the evolution of the above described pair of interacting quantum dots:

$$
\frac{d \rho}{d t}=\operatorname{tr}_{\mathrm{Ph}}\left(-\frac{\mathrm{i}}{\hbar}\left[H, \rho_{\mathrm{tot}}\right]\right), \quad \text { with } \rho_{\mathrm{tot}}(0)=\rho(0) \otimes \frac{e^{-\beta H_{\mathrm{Ph}}}}{\operatorname{tr}\left(e^{-\beta H_{\mathrm{Ph}}}\right)} .
$$

\subsection{Measure of entanglement}

To study the coherence properties of the system we used the definition of the entanglement of formation as a measure of entanglement. The entanglement of a bipartite pure state is defined as an entropy of either of its subsystems. For the mixed states the definition of entanglement is generalized to the entanglement of formation. For a mixed state of a bipartite system the entanglement of formation is defined as the minimal possible entanglement over all the quantum ensembles representing the mixed state $[19,31]$. The entanglement of formation of the density matrix is usually evaluated in the Bell basis

$$
\left|e_{1}\right\rangle=\frac{1}{\sqrt{2}}(|X X\rangle+|00\rangle),\left|e_{2}\right\rangle=\frac{\mathrm{i}}{\sqrt{2}}(|X X\rangle-|00\rangle),\left|e_{3}\right\rangle=\frac{\mathrm{i}}{\sqrt{2}}(|X 0\rangle+|0 X\rangle),\left|e_{4}\right\rangle=\frac{1}{\sqrt{2}}(|X 0\rangle-|0 X\rangle),
$$

using the following procedure [19,31]. The four eigenvalues $\lambda_{1} \geq \lambda_{2} \geq \lambda_{3} \geq \lambda_{4}$ of the auxiliary matrix

$$
R(\rho)=\sqrt{\sqrt{\rho} \rho^{*} \sqrt{\rho}}
$$

where $\rho^{*}$ denotes the complex conjugation, are used to evaluate the concurrence $C=\max \left(0, \lambda_{1}-\lambda_{2}-\right.$ $\lambda_{3}-\lambda_{4}$ ). The entanglement of formation is then given by

$$
E(\rho)=H\left(\frac{1}{2}+\frac{1}{2} \sqrt{1-C^{2}}\right), \quad \text { where } H(x)=-x \log _{2} x-(1-x) \log _{2}(1-x) .
$$

The entanglement of the singlet state is exactly one.

\subsection{Numerical calculation of the path integral}

A C ++ code was designed for the evalution of the density matrices of systems interacting with a heat bath according to the method described in [21]. The total evolution time $t$ is divided into $N$ time slices $t_{n}=\epsilon n, \epsilon=t / N$. The final density matrix at time $t_{N}=t$ is given by

$$
\rho_{\alpha_{N}, \beta_{N}}=\exp \left[\mathrm{i} t\left(\hat{\Omega}_{\beta_{N} \beta_{N}}-\hat{\Omega}_{\alpha_{N} \alpha_{N}}\right)\right] \sum_{\left\{\alpha_{n}, \beta_{n}\right\}} \prod_{n=1}^{N} M_{\alpha_{n}}^{\alpha_{n-1}} M_{\beta_{n-1}}^{\beta_{n^{*}}} \prod_{n^{\prime}=1}^{n} \exp \left(S_{n n^{\prime}}\right) \rho_{\alpha_{0} \beta_{0}},
$$

where $\hat{\Omega}=\operatorname{diag}(0, \delta, \delta, 2 \delta)$ is the diagonal part of the system Hamiltonian without bath. The evaluation is performed using the method of augmented density matrix evaluation [29, 30]:

$$
\bar{R}_{n}=T_{n} \bar{R}_{n-1},
$$

where the augmented density matrix $\bar{R}_{n}$ coincides with the density matrix of the system $R_{n}$ for all discrete time instants less or equal to the memory length $n_{c}$, or is truncated by the last time instant 
$n-n_{c}-1$. This means that up to the moment $n=n_{c}$ we have an iteration procedure which increases the path length by one at each step,

$$
R_{n}=T_{n} R_{n-1}, \quad n \leq n_{c} .
$$

Starting from the moment $n=n_{c}+1$, all the paths with coinciding states except for the earliest time state at $n-n_{c}-1$ are summed up over that state to keep the non-increasing record length of $n_{c}$ time instants.

The iteration operator has the form:

$$
T_{n}=M_{\alpha_{n}}^{\alpha_{n-1}} M_{\beta_{n-1}}^{\beta_{n^{*}}} \exp \left\{\sum_{n^{\prime}=\max \left(1, n-n_{c}\right)}^{n} S_{n n^{\prime}}\right\} .
$$

The system rotation operator has the form $M_{\alpha_{n}}^{\alpha_{n-1}} \equiv\left\langle\alpha_{n}\left|e^{-\mathrm{i} \epsilon \hat{M}\left(t_{n}\right)}\right| \alpha_{n-1}\right\rangle \equiv M\left(\alpha_{n}, \alpha_{n-1}\right)$, where $\alpha, \beta=\overline{0,3}$ are the indexes of the quantum state of the system. For the two qubits these states are $00,0 X, X 0, X X$.

The matrix $\hat{M}$ is the non-diagonal part of the system Hamiltonian. For the pair of qubits with dipole-dipole interaction we write

$$
\hat{M}=\left(\begin{array}{cccc}
0 & 0.5 K_{1} & 0.5 K_{2} & 0.0 \\
0.5 K_{1} & 0 & J_{12} & 0.5 K_{2} \\
0.5 K_{2} & J_{21} & 0 & 0.5 K_{1} \\
0 & 0.5 K_{2} & 0.5 K_{1} & 0
\end{array}\right) .
$$

At the initial instant of time $n=0$ the augmented density matrix is equal to the initial density matrix of the system: $R_{0}=\bar{\rho}_{\alpha_{0} \beta_{0}}(0), \alpha_{n}, \beta_{n}$ are the time forward (backward) state variables. $\gamma=(0,1,1,2)$ is the coupling of the states $(00, \mathrm{X} 0,0 \mathrm{X}, \mathrm{XX})$ to phonons. The 'action' $S_{n n^{\prime}}$ is given by the equation (A34) of [21]: $S_{n n^{\prime}}=-K_{\alpha_{n}^{\prime} \alpha_{n}}-K_{\beta_{n}^{\prime} \beta_{n}}^{\dagger}+K_{\beta_{n}^{\prime} \alpha_{n}}^{\dagger}+K_{\alpha_{n}^{\prime} \beta_{n}}$. For real-valued phonon coupling this gives

$$
S_{n \neq n^{\prime}}=\left(\gamma\left(\alpha_{n}^{\prime}\right)+\gamma\left(\beta_{n}^{\prime}\right)\right)\left(\gamma\left(\beta_{n}\right)-\gamma\left(\alpha_{n}\right)\right) K\left(n^{\prime}-n\right), \quad S_{n n}=\left(\gamma\left(\beta_{n}\right)-\gamma\left(\alpha_{n}\right)\right)\left(\gamma\left(\alpha_{n}\right) K(0)-\gamma\left(\beta_{n}\right) K^{*}(0)\right) \text {. }
$$

The function $K(m, \epsilon, \beta=1 / T)$ is given by

$$
\begin{aligned}
K_{m \neq 0} & =\int_{m \epsilon}^{(m+1) \epsilon} d \tau \int_{0}^{\epsilon} d \tau^{\prime} \Gamma\left(\tau-\tau^{\prime}\right)= \\
& =2 \int_{0}^{\infty} d \omega \frac{J(\omega)}{\omega^{2}}[1-\cos (\omega \epsilon)]\{\cos (\omega \epsilon m) \operatorname{coth}(\omega \beta / 2)-\mathrm{i} \sin (\omega \epsilon m)\}, \\
K_{m=0} & =\int_{0}^{\epsilon} d \tau \int_{0}^{\tau} d \tau^{\prime} \Gamma\left(\tau-\tau^{\prime}\right)= \\
& =\int_{0}^{\infty} d \omega \frac{J(\omega)}{\omega^{2}}\{[1-\cos (\omega \epsilon)] \operatorname{coth}(\omega \beta / 2)-\mathrm{i} \epsilon \omega+\mathrm{i} \sin (\epsilon \omega)\}
\end{aligned}
$$

\section{Results and Conclusions}

We have performed the simulation for two different sizes of QDs: a) $d=3.3 \mathrm{~nm}$ and b) $d=33 \mathrm{~nm}$. The interdot distance was set to $L=10 \mathrm{~nm}$ and $L=50 \mathrm{~nm}$, for this two cases, respectively. The initial density matrix in our simulation correspond to the symmetric entangled state of two QDs, which is $\left|e_{3}\right\rangle\left\langle e_{3}\right|$ in the magic basis (4). The corresponding density matrix in the standard basis $00,0 X, X 0, X X$ is given by

$$
\rho(0)=\left(\begin{array}{cccc}
0 & 0 & 0 & 0 \\
0 & 0.5 & 0.5 & 0 \\
0 & 0.5 & 0.5 & 0 \\
0 & 0 & 0 & 0
\end{array}\right)
$$


The parameters of InGaAs/GaAs QDs were taken in accordance to $[9,20]$. These are $m_{*}=0.067 m_{e}$, $\rho_{\mathrm{GaAs}}=5.37 \mathrm{~g} / \mathrm{cm}^{3}, v_{s}=5.11 \cdot 10^{5} \mathrm{~cm} / \mathrm{s}, \epsilon=10.0, a_{0}=3.94 \mathrm{~nm}$. The dipole-dipole coupling constant was estimated as $J=\mu^{2} /\left(\varepsilon L^{3}\right)$, where $\mu$ is the transition dipole moment between the ground and the first excited states of the quantum dot. For the above cases a) and b) these values are $J=0.595 \mathrm{ps}^{-1}$ and $J=0.476 \mathrm{ps}^{-1}$ respectively. The value of the driving field was taken from [32] and corresponds to $1.9 \mathrm{kV} / \mathrm{cm}$. The cut-off frequencies are a) $\omega_{c}=2.2 \mathrm{ps}^{-1}$ and b) $\omega_{c}=0.22 \mathrm{ps}^{-1}$ in accordance to [20]. Being written in the magic basis (4) the asymptotics of evolution shown in Figure 1 corresponds

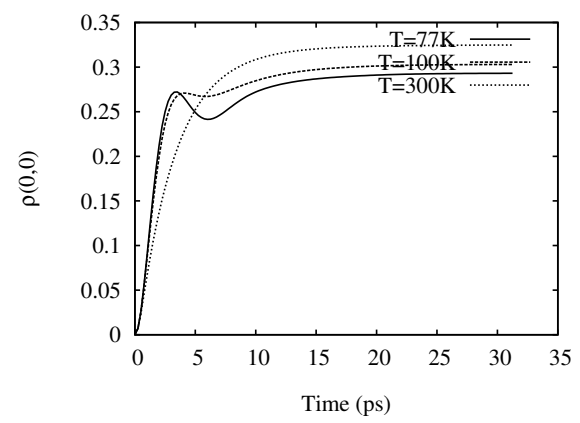

a) $d=3.3 \mathrm{~nm}, L=10 \mathrm{~nm}$

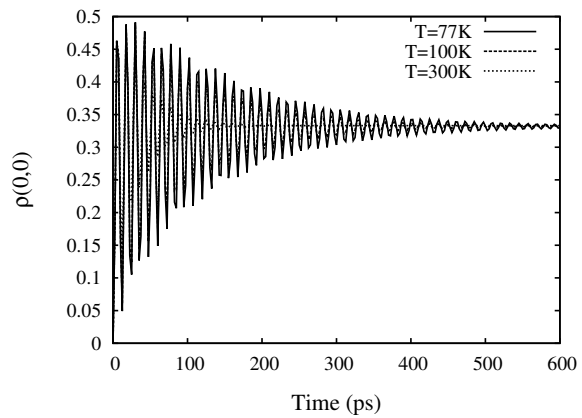

b) $d=33 \mathrm{~nm}, L=50 \mathrm{~nm}$

Figure 1. Evolution of the density matrix element $\rho(0,0)$ with the initial condition (14) for a) $d=3.3 \mathrm{~nm}$, $\mu=79.3$ Debye and b) $d=33 \mathrm{~nm}, \mu=793$ Debye for InGaAs/GaAs QDs

to the spread of the state $e_{3}$ into the equally weighted triplet $\left(e_{1}, e_{2}, e_{3}\right)$. The asymptotic value of the density matrix $\rho$ written in the magic basis is $\rho(+\infty)=\operatorname{diag}(1 / 3,1 / 3,1 / 3,0)$.

The graph of the entanglement of formation corresponding to this evolution is shown in figure 2 below. The simulation above shows the robustness of this time evolution with respect to the bath

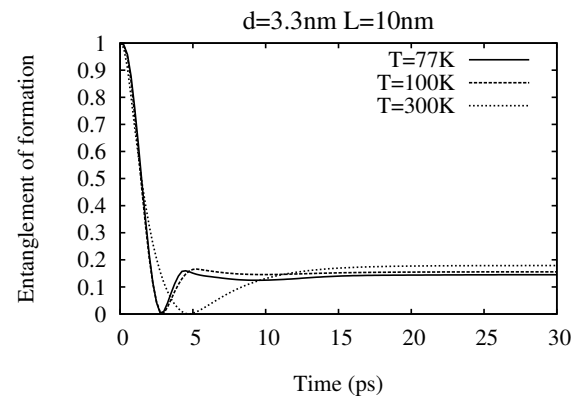

a)

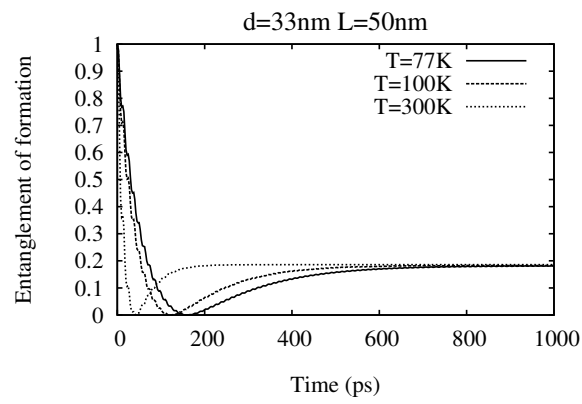

b)

Figure 2. Time dependence of entanglement of formation calculated with the initial condition (14): a) $d=3.3 \mathrm{~nm}$ and b) $d=33 \mathrm{~nm}$ InGaAs/GaAs QDs; $n_{c}=3$ in these simulations and some non-Markoff effects might be lost

temperature parameter $T=77 \div 300 \mathrm{~K}$ and the existence of attractors in the space of the density matrices. 


\section{Acknowledgements}

The work was supported in part by RFBR projects 13-07-00409, 14-02-00739 and by the Ministry of Education and Science of the Russian Federation in the framework of Increase Competitiveness Program of "MISIS".

\section{References}

[1] S. Kak, Inf. Sci. 83, 143 (1995)

[2] M. Altaisky, N. Kaputkina, V. Krylov, Physics of Particles and Nuclei 45, 1013 (2014)

[3] E. Cohen, B. Tamir, Int. J. Quant. Inf. 12, 1430002 (2014)

[4] P. Rebentrost, M. Mohseni, S. Lloyd, Phys. Rev. Lett. 113, 130503 (2014)

[5] M. Schuld, I. Sinayskiy, F. Petruccione, Quantum Information Processing 13, 2567 (2014)

[6] Johnson M. W., Amin M. H. S., Gildert S., Lanting T., Hamze F., Dickson N., Harris R., Berkley A. J., Johansson J., Bunyk P. et al., Nature 473, 194 (2011)

[7] V. Smelianskiy, a.o., arxiv.org:1204.2821 (2012)

[8] A. Ramsay, A. Fox, M. Skolnik, A. Gopal, E. Gauger, B. Lovett, A. Nazir, Phys. Rev. Lett. 104, $017402(2010)$

[9] A.J. Ramsay, T.M. Godden, S.J. Boyle, E.M. Gauger, A. Nazir, B.W. Lovett, A.M. Fox, M.S. Skolnik, Phys. Rev. Lett. 105, 177402 (2010)

[10] C. Sikorski, U. Merkt, Phys. Rev. Lett. 62, 2164 (1989)

[11] A. Kumar, S.E. Laux, F. Stern, Phys. Rev. B 42, 5166 (1990)

[12] M. Wagner, U. Merkt, A. Chaplik, Phys. Rev. B 45, 1951 (1995)

[13] R.C. Ashoori, H.L. Stormer, J.S. Weiner, L.N. Pfeiffer, K.W. Baldwin, K.W. West, Phys. Rev. Lett. 71, 613 (1993)

[14] H. Drexler, D. Leonard, W. Hansen, J.P. Kotthaus, P.M. Petroff, Phys. Rev. Lett. 73, 2252 (1994)

[15] Y.M. Blanter, N. Kaputkina, Y.E. Lozovik, Physica Scripta 54, 539 (1996)

[16] D. Loss, D. DiVincenzo, Phys. Rev. A 57, 120 (1998)

[17] E. Behrman, L. Nash, J. Steck, V. Chandrashekar, S. Skinner, Inf. Sci. 128, 257 (2000)

[18] A. Balandin, D. Nika, Materials Today 15, 266 (2012)

[19] C. Bennet, D. DiVincenzo, J. Smolin, W. Wooters, Phys. Rev. A 54, 3824 (1996)

[20] A. Nazir, Phys. Rev. B 78, 153309 (2008)

[21] A. Vagov, M.D. Croitoru, M. Glässl, V.M. Axt, T. Kuhn, Phys. Rev. B 83, 094303 (2011)

[22] D. McCutcheon, N. Dattani, E. Gauger, B. Lovett, A. Nazir, Phys. Rev. B 84, 081305(R) (2011)

[23] B. Coffey, R. Friedberg, Phys. Rev. A 17, 1033 (1978)

[24] S. John, T. Quang, Phys. Rev. A 52, 4083 (1995)

[25] F.J. Rodríguez, L. Quiroga, N.F. Johnson, Phys. Rev. B 66, 161302 (2002)

[26] N. Dattani, AIP Advances 2, 012121 (2012)

[27] N. Dattani, F. Pollock, D. Wilkins, Quantum Physics Letters 1, 35 (2012)

[28] N. Dattani, Comp. Phys. Comm. 184, 2828 (2013)

[29] N. Makri, D. Makarov, Journal of Chemical Physics 102, 4600 (1995)

[30] N. Makri, D. Makarov, Journal of Chemical Physics 102, 4611 (1995)

[31] S. Hill, W. Wootters, Phys. Rev. Lett. 78, 5022 (1997)

[32] G.Y. Slepyan, A. Magyarov, S.A. Maksimenko, A. Hoffmann, Phys. Rev. B 76, 195328 (2007) 